\title{
Infected Iliac Artery Aneurysm Concomitant with Liver Abscess Caused by Fusobacterium nucleatum
}

\author{
Satoshi Yamamoto*, Takafumi Akai, Takehiro Chiyoda, Hiroshi Goto, Yukiyoshi Masaki \\ Department of Surgery, Ome Municipal General Hospital, Tokyo, Japan \\ Email: "syamamoto-tky@umin.ac.jp
}

Received 30 June 2016; accepted 22 July 2016; published 25 July 2016

Copyright (C) 2016 by authors and Scientific Research Publishing Inc.

This work is licensed under the Creative Commons Attribution International License (CC BY). http://creativecommons.org/licenses/by/4.0/

(c) (i) Open Access

\begin{abstract}
We report a case of infected iliac artery aneurysm concomitant with liver abscesses caused by Fusobacterium nucleatum. A 58-year-old man developed an aneurysm of the right common iliac artery and liver abscesses. The aneurysm was resected and a femoro-femoral crossover bypass with a knitted Dacron graft was performed for impending rupture. Anaerobic cultures obtained from blood and intramural thrombus were positive for Fusobacterium nucleatum. With antibiotics, the liver abscesses disappeared without drainage. Iliopsoas abscesses developed after surgery, but it was controlled with antibiotics. The patient was free of infection 1 year after the surgery. The causative bacterium was suspected to originate in the oral cavity, because the patient had a notable history of poor chronic periodontal conditions. Clinically, infected aortoiliac aneurysm complicated by Fusobacterium is extremely rare relative to the prevalence of the pathogenic bacterium. However, it is noteworthy that Fusobacterium can cause this condition.
\end{abstract}

\section{Keywords}

Infected Aortoiliac Aneurysm, Fusobacterium nucleatum, Liver Abscess

\section{Introduction}

Infected aortoiliac aneurysm (IAA) is rare, but can be fatal due to rupture and sepsis [1]-[3]. Appropriate antibiotic is mandatory for the treatment of IAA. However, culture studies are often negative and the causative bacterium is often unidentified [3] [4]. It is necessary to consider possible pathogenic bacteria in such a case. The pathogenic bacteria causing IAA are usually Salmonella, Staphylococcus, and Streptococcus; other species includ-

${ }^{*}$ Corresponding author.

How to cite this paper: Yamamoto, S., Akai, T., Chiyoda, T., Goto, H. and Masaki, Y. (2016) Infected Iliac Artery Aneurysm Concomitant with Liver Abscess Caused by Fusobacterium nucleatum. Case Reports in Clinical Medicine, 5, 238-242. 
ing Bacteroides have been reported to complicate IAA [3] [5]-[7]. However, clinically, Fusobacterium is extremely rare as a causative bacterium of IAA [8], although periodontal pathogens are often identified in aortoiliac aneurysms and Fusobacterium is a periodontal pathogen that can spread to extra-oral sites [9]-[13]. Additionally, liver abscess caused by Fusobacterium is rare [14] [15]. We report a case of IAA concomitant with liver abscess caused by F. nucleatum, which was suspected to originate in the oral cavity.

\section{Case Report}

A 58-year-old man was emergently admitted to our hospital complaining of a four-day history of right lower quadrant pain. Four weeks prior to admission, he developed a fever of $40^{\circ} \mathrm{C}$, and had intermittent high fevers over $38^{\circ} \mathrm{C}$. The patient's past medical history included right hemifacial spasm, carious teeth, and gingival swelling. He often experienced shedding of the dental crown, the last episode of which occurred 3 months prior to admission. He did not have diabetes mellitus or immune deficiency. He was not taking steroids or immunosuppressants. He was a former smoker but was not an alcoholic. His vital signs on admission were as follows: temperature, $37.8^{\circ} \mathrm{C}$; heart rate, 117 beats $/ \mathrm{min}$; blood pressure, 133/81 mm Hg; and respiratory rate, 22 breaths $/ \mathrm{min}$. Physical examination showed a pulsatile mass in the right lower quadrant of the abdomen. Blood tests revealed the following values: white blood cell count, 25,800 cells $/ \mathrm{mm}^{3}$; hemoglobin, $10.2 \mathrm{~g} / \mathrm{dL}$; C-reactive protein, 29.8 $\mathrm{mg} / \mathrm{dL}$; total bilirubin, $1.3 \mathrm{mg} / \mathrm{dL}$; aspartate aminotransferase, $159 \mathrm{IU} / \mathrm{L}$; alanine aminotransferase, $141 \mathrm{IU} / \mathrm{L}$; lactate dehydrogenase, 309 IU/L; alkaline phosphatase, $1144 \mathrm{IU} / \mathrm{L}$; and gamma glutamyl transpeptidase, 191 IU/L. Computed tomography (CT) showed an aneurysm of the right common iliac artery (CIA) and liver abscesses (Figure 1(a) and Figure 2(a)). Emergency surgery was performed for impending rupture of the aneurysm. We performed a femoro-femoral crossover bypass with a knitted Dacron graft coated with rifampicin and resected the aneurysm (Figure 1(b)). The aneurysm had malodorous contents, and we removed the infected tissues and thrombus. The resected site was irrigated adequately with saline water, and the stumps of the arteries were covered with the omentum.

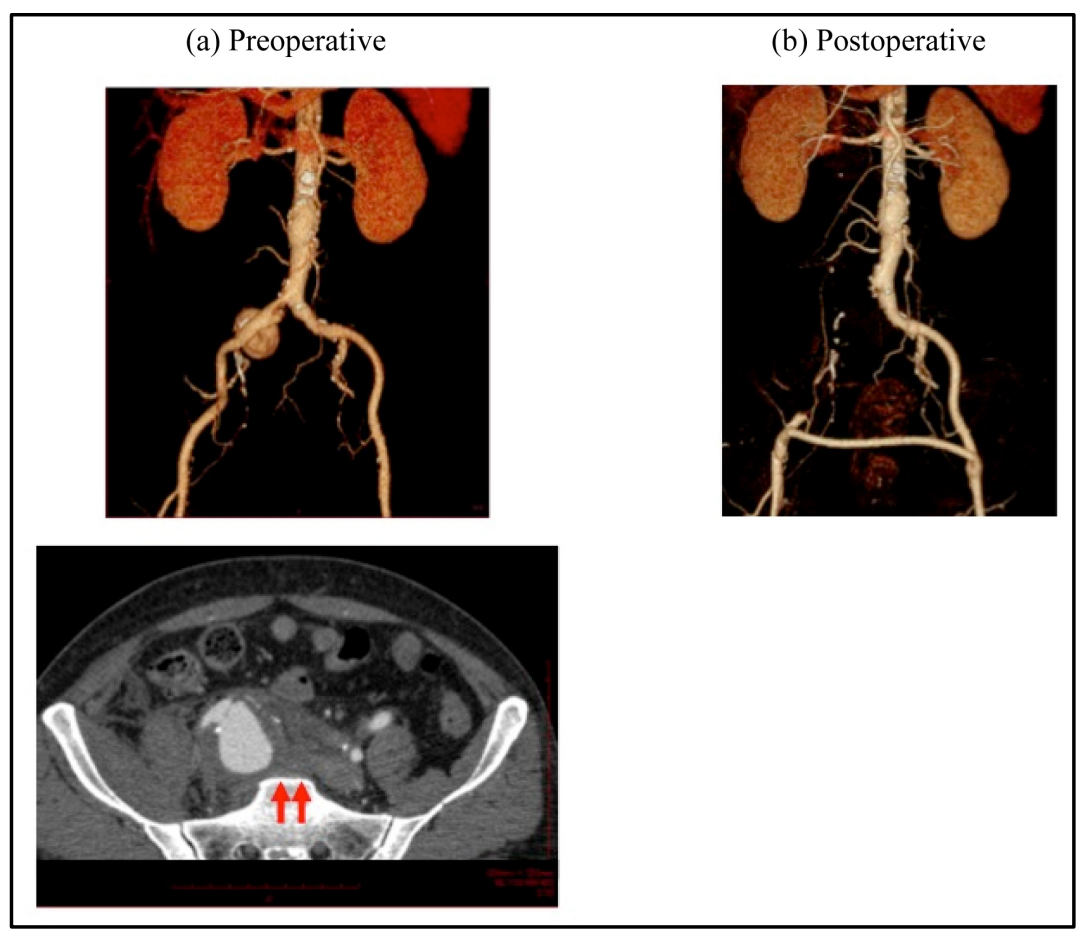

Figure 1. Computed tomography (CT) of the right common iliac artery (CIA) aneurysm. Preoperative CT shows a saccular aneurysm of the right CIA $4 \mathrm{~cm}$ in diameter (a). The aneurysm has irregular posterior contour (a, red arrows). The other aortoiliac arteries show atherosclerotic changes with calcification. Postoperative CT shows that the CIA aneurysm has been resected and the bypass graft between the femoral arteries is patent (b). 
Postoperatively, cultures obtained from blood and intramural thrombus proved to be positive for F. nucleatum. Echocardiography did not show any evidence of infective endocarditis (IE). Magnetic resonance imaging (MRI) and magnetic resonance angiography (MRA) did not detect abscess or aneurysm in craniocervical sites. The patient was treated with doripenem $3 \mathrm{~g} /$ day for 7 days after the surgery, and with clindamycin $2.4 \mathrm{~g} /$ day for the next 17 days on the basis of the antibiotic sensitivity. According to the treatment with the antibiotics, the infection was controlled and postoperative blood culture studies were negative. Postoperative CT revealed that the liver abscesses diminished without drainage on day 6 after surgery (Figure 2(b)). Postoperative CT detected the occurrence of right iliopsoas abscesses on day 6 after surgery (Figure 3(a)), but the abscesses diminished on day

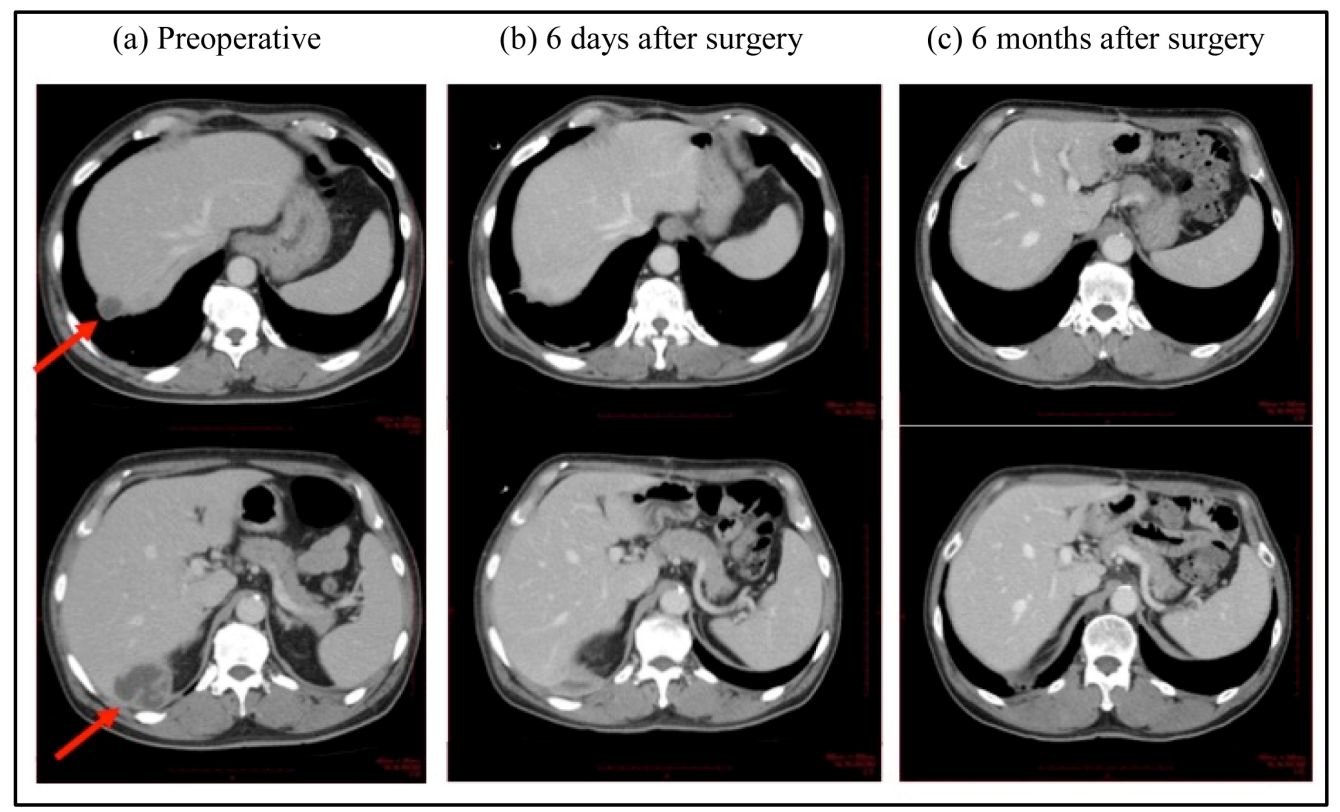

Figure 2. Computed tomography (CT) of the liver abscesses. Preoperative CT shows the liver abscesses in 2 segments of S6 and S7 ((a), red arrows; $4.5 \mathrm{~cm}$ and $2.0 \mathrm{~cm}$ in size, respectively). Postoperative CT shows that the liver abscesses diminished 6 days after surgery (b) and have almost disappeared 6 months after surgery (c).

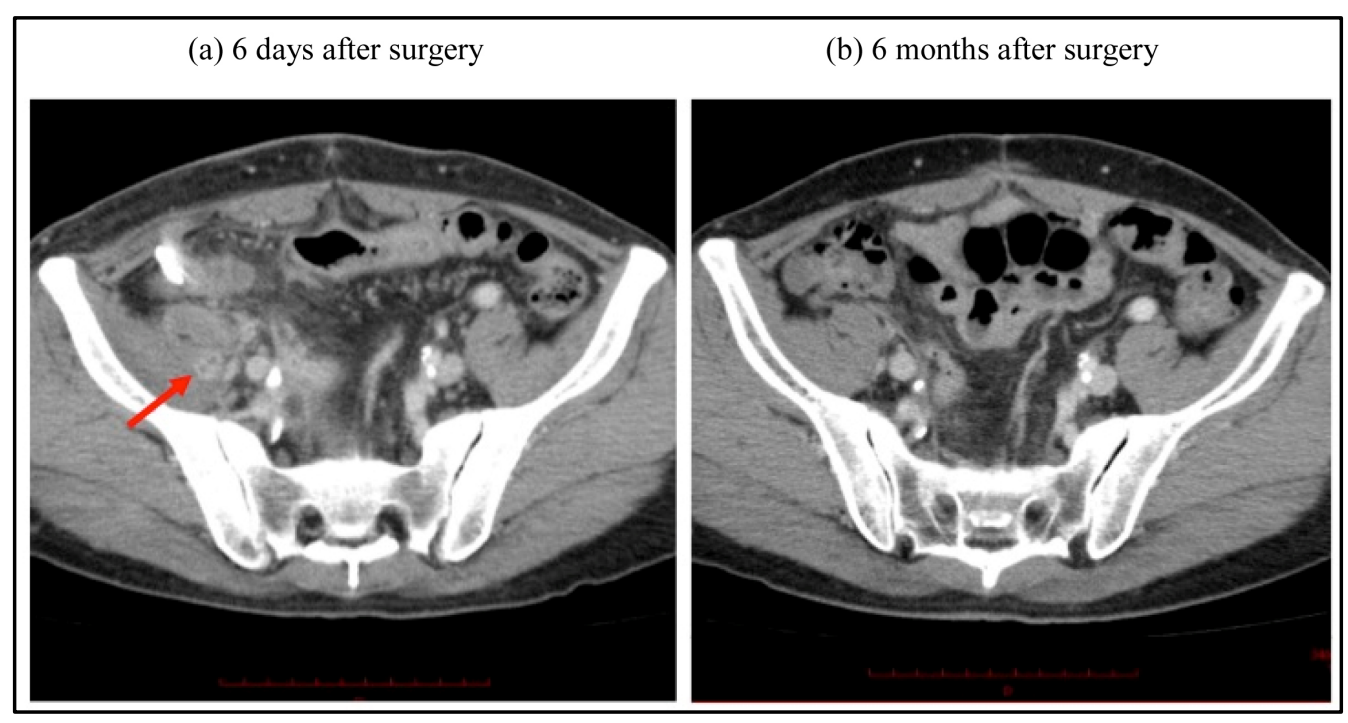

Figure 3. Computed tomography (CT) of the iliopsoas abscesses. Postoperative CT shows the emergence of the iliopsoas abscesses 6 days after surgery ((a), red arrow), but the abscesses have disappeared 6 months after surgery (b). 
24 after surgery. The patient began to take clindamycin $0.9 \mathrm{~g} /$ day orally on day 25 after the surgery and was discharged on day 29 after surgery. Sultamicillin replaced clindamycin because of neutropenia induced by clindamycin 6 weeks after surgery. CT revealed no signs of recurrence of infection and the abscesses of the liver and psoas muscle had almost disappeared 6 months after surgery (Figure 2(c) and Figure 3(b)). At 1-year follow-up, the bypass graft was patent and the patient remained free of infection.

\section{Discussion}

The patient was diagnosed with infected CIA aneurysm caused by F. nucleatum on the basis of clinical signs of infection (fever, pain, and leukocytosis) and positive cultures obtained from blood and intramural thrombus [2] [3]. IAA has been classified into the following four types: mycotic aneurysms, microbial arteritis with aneurysm, infected preexisting aneurysms, and posttraumatic infected false aneurysms [5]. In our case, the aneurysm was not likely to be a mycotic aneurysm derived from IE. A posttraumatic infected false aneurysm was not likely because the patient had no recent history of vascular injury due to trauma or medical procedure. The false saccular aneurysm did not have calcified aneurysmal walls that indicated a preexisting aneurysm, while the other aortoiliac arteries had arteriosclerotic changes. Therefore, we considered a microbial arteritis with aneurysm rather than an infected preexisting aneurysm. As the source of infection of the CIA, it is improbable that the nearby iliopsoas abscesses spread directly, because the abscesses appeared after surgery. It is possible that the bacterium infected the CIA from the liver abscess in the event that the occurrence of the liver abscess was primary. Because Fusobacterium species commensally inhabit the gastrointestinal tract as well as the oropharyngeal cavity, the bacterium could be from the gastrointestinal tract. However, we surmise that the bacterium originated in the oral cavity, because periodontal pathogens are often identified in atherosclerotic lesions and aortoiliac aneurysms [9] [12] [13]; the patient had no gastrointestinal diseases, but had a notable history of poor chronic periodontal conditions.

F. nucleatum is one of the most prevalent species in extra-oral sites under disease conditions [9]-[11]. F. nucleatum is frequently associated with complications of the head and neck, such as thrombophlebitis of the jugular vein (Lemierre's syndrome) and ruptured cerebral aneurysm [10]. In our case, MRI and MRA did not detect any lesions in the head and neck, but CT showed abscesses in the liver and iliopsoas muscle. Although those abscesses were not sampled, F. nucleatum was probably the causative bacterium of the abscesses on the basis of the culture studies. We assume that bacteremia with $F$. nucleatum caused the abscesses, although it is uncertain whether the infection of the CIA was primary to the liver abscesses. The most frequent bacteria cultured from liver abscesses are Klebsiella pneumoniae, Escherichia coli, and Streptococcus spp., and liver abscess caused by Fusobacterium spp. is rare [14]-[16]. Several cases of IAA associated with liver abscess have been reported, but they were not caused by Fusobacterium [17] [18]. Therefore, IAA concomitant with liver abscess caused by Fusobacterium is extremely rare. To our knowledge, this is the second case of IAA caused by Fusobacterium, and the first case of IAA concomitant with liver abscess caused by Fusobacterium. The reason for the rarity of IAA caused by Fusobacterium compared to other anaerobic bacteria such as Bacteroides is unclear, but we assume that differences exist in the tendencies of bacterial species to infect arteriosclerotic lesions or aneurysms. Meanwhile, the true prevalence of IAA caused by Fusobacterium may be higher than previously reported because the bacterium might have been missed in some cases in which the culture study was negative. We surmise that there are instances in which anaerobic culture was not performed, routine anaerobic culture failed to detect the bacterium due to the difficulty in cultivation, or the bacterium had disappeared with antibiotics at the time of sampling.

For IAA, it is advisable to control bacterial infection with antibiotics before surgical treatment [2]. However, emergency surgery was performed, because the impending rupture of the aneurysm was evident on arrival at the hospital. In situ reconstruction is considered a safe and durable alternative [3], but in such cases, controlling the infection control is often difficult when it is recurrent. We performed an extra-anatomical bypass, because we had considered the risk of residual infection due to rupture and the possibility of relaparotomy for the liver abscesses. For liver abscess, percutaneous drainage had a risk of injury to the lung or to the intestinal tract. Simultaneous open surgical drainage had a risk of dissemination of the bacterium into the peritoneal cavity, which would make infection control difficult. We were considering drainage for the liver abscesses after the emergency surgery for IAA, but the abscesses reduced with antibiotic therapy. 


\section{Conclusion}

IAA caused by Fusobacterium is rare. However, it is necessary to consider the possibility of a periodontal pathogen including Fusobacterium as a causative bacterium in patients with IAA. Anaerobic culture study is mandatory for the treatment of IAA.

\section{References}

[1] Gomes, M.N., Choyke, P.L. and Wallace, R.B. (1992) Infected Aortic Aneurysms. A Changing Entity. Annals of Surgery, 215, 435-442. http://dx.doi.org/10.1097/00000658-199205000-00005

[2] Muller, B.T., Wegener, O.R., Grabitz, K., Pillny, M., Thomas, L. and Sandmann, W. (2001) Mycotic Aneurysms of the Thoracic and Abdominal Aorta and Iliac Arteries: Experience with Anatomic and Extra-Anatomic Repair in 33 Cases. Journal of Vascular Surgery, 33, 106-113. http://dx.doi.org/10.1067/mva.2001.110356

[3] Oderich, G.S., Panneton, J.M., Bower, T.C., et al. (2001) Infected Aortic Aneurysms: Aggressive Presentation, Complicated Early Outcome, But Durable Results. Journal of Vascular Surgery, 34, 900-908. http://dx.doi.org/10.1067/mva.2001.118084

[4] Ernst, C.B., Campbell Jr., H.C., Daugherty, M.E., Sachatello, C.R. and Griffen Jr., W.O. (1977) Incidence and Significance of Intra-Operative Bacterial Cultures during Abdominal Aortic Aneurysmectomy. Annals of Surgery, 185, 626633. http://dx.doi.org/10.1097/00000658-197706000-00003

[5] Weaver, M.R. and Reddy, D.J. (2010) Infected Aneurysms. In: Cronenwett, J.L. and Johnston, K.W., Eds., Rutherford's Vascular Surgery, 7th Edition, Saunders, Philadelphia, 2156-2167. http://dx.doi.org/10.1016/b978-1-4160-5223-4.00139-6

[6] Hsu, R.B., Chen, R.J., Wang, S.S. and Chu, S.H. (2004) Infected Aortic Aneurysms: Clinical Outcome and Risk Factor Analysis. Journal of Vascular Surgery, 40, 30-35. http://dx.doi.org/10.1016/j.jvs.2004.03.020

[7] O'Donnell, J.A. and Asbel, L.E. (1999) Bacteroides Fragilis Bacteremia and Infected Aortic Aneurysm Presenting as Fever of Unknown Origin: Diagnostic Delay without Routine Anaerobic Blood Cultures. Clinical Infectious Diseases, 29, 1309-1311. http://dx.doi.org/10.1086/313429

[8] Wotherspoon, D., Street, J.A., Hedderwick, S. and Baker, R. (2012) Fusobacterium Necrophorum in an Abdominal Aortic Aneurysm, Treated by Once Daily Ertapenem. International Journal of Angiology, 21, 175-176. http://dx.doi.org/10.1055/s-0032-1325169

[9] Armingohar, Z., Jorgensen, J.J., Kristoffersen, A.K., Abesha-Belay, E. and Olsen, I. (2014) Bacteria and Bacterial DNA in Atherosclerotic Plaque and Aneurysmal Wall Biopsies from Patients with and without Periodontitis. Journal of Oral Microbiology, 6, 23408. http://dx.doi.org/10.3402/jom.v6.23408

[10] Han, Y.W. (2015) Fusobacterium Nucleatum: A Commensal-Turned Pathogen. Current Opinion in Microbiology, 23, 141-147. http://dx.doi.org/10.1016/j.mib.2014.11.013

[11] Han, Y.W. and Wang, X.(2013) Mobile Microbiome: Oral Bacteria in Extra-Oral Infections and Inflammation. Journal of Dental Research, 92, 485-491. http://dx.doi.org/10.1177/0022034513487559

[12] Leishman, S.J., Do, H.L. and Ford, P.J. (2010) Cardiovascular Disease and the Role of Oral Bacteria. Journal of Oral Microbiology, 2, 5781. http://dx.doi.org/10.3402/jom.v2i0.5781

[13] Suzuki, J., Aoyama, N., Aoki, M., et al. (2014) High Incidence of Periodontitis in Japanese Patients with Abdominal Aortic Aneurysm. International Heart Journal, 55, 268-270. http://dx.doi.org/10.1536/ihj.13-301

[14] Nagpal, S.J., Mukhija, D. and Patel, P. (2015) Fusobacterium Nucleatum: A Rare Cause of Pyogenic Liver Abscess. Springerplus, 4, 283. http://dx.doi.org/10.1186/s40064-015-1090-8

[15] Buelow, B.D., Lambert, J.M. and Gill, R.M. (2013) Fusobacterium Liver Abscess. Case Reports in Gastroenterology, 7, 482-486. http://dx.doi.org/10.1159/000356821

[16] Heneghan, H.M., Healy, N.A., Martin, S.T., et al. (2011) Modern Management of Pyogenic Hepatic Abscess: A Case Series and Review of the Literature. BMC Research Notes, 4, 80. http://dx.doi.org/10.1186/1756-0500-4-80

[17] Beltran-Ordonez, I., Yian, T., Hou, A. and Hai, E. (2015) Mycotic Aneurysm of Infrarenal Aorta: A Case Report and Review of Literature. Journal of Biosciences and Medicines, 3, 88-93. http://dx.doi.org/10.4236/jbm.2015.33013

[18] Youn, J.K., Kim, S.M., Han, A., et al. (2015) Surgical Treatment of Infected Aortoiliac Aneurysm. Vascular Specialist International, 31, 41-46. http://dx.doi.org/10.5758/vsi.2015.31.2.41 


\section{Submit or recommend next manuscript to SCIRP and we will provide best service for you:}

Accepting pre-submission inquiries through Email, Facebook, LinkedIn, Twitter, etc.

A wide selection of journals (inclusive of 9 subjects, more than 200 journals)

Providing 24-hour high-quality service

User-friendly online submission system

Fair and swift peer-review system

Efficient typesetting and proofreading procedure

Display of the result of downloads and visits, as well as the number of cited articles

Maximum dissemination of your research work

Submit your manuscript at: http://papersubmission.scirp.org/ 